\title{
Production of grazed tropical grasses in different agroecosystems in Puerto Rico. II. humid northern coastal plains ${ }^{1}$
}

\author{
Lais E. Tergas, Jaime Vélez-Santiago and Doralisa Vera de Saldaña
}

\begin{abstract}
A field experiment was conducted near Barceloneta in the humid northern coastal plains of Puerto Rico to evaluate the production and persistence of 10 tropical grasses: Cynodon sp. Local, Digitaria pentzii Slenderstem, $\boldsymbol{C}$. nlemfuensis Star, C. plectostachyus Star, C. dactylon Coastcross-1, D. milanijana Pangola Soto, Panicum maximum Guinea, P. maximum Guinea USDA PI 259553, $P$. maximum Makueni and $D$. decumbens Transvala grazed at 3- to 5-week intervals for one and a half year, $P$. maximum USDA PI 259553 was the most productive grass, but during the 13 grazings in 1981-1982, it was not significantly different $(P=0.05)$ from $C$. plectos: tachyus Star; Cynodon sp. Local, D. pentzii Slenderstem, C. nlemfuensis Star, $P$. maximum Makueni, and $D$. decumbens Transvala. Guinea and $D$. milaniana Pangola Soto were the least productive $(P=0.05)$. Results for 7 grazings from January to June 1983 again showed $P$. maximum USDA PI $\mathbf{2 5 9 5 5 3}$ to be the most productive grass, although not significantly different $(P=0.05)$ from $D$. decumbens Transvala, $C$. dactylon Coastcross -1 and $D$. pentzii. The production of $P$. maximum Makueni and $C$. plectostachyus Star was intermediate; $C$. nlemfuensis Star and Guinea were the least productive, and D. milaniiana Pangola Soto did not persist under intensive grazing $(P=0.05)$. The total mean production of all grasses of 0.92 and $1.00 \mathrm{ton} / \mathrm{ha} / \mathrm{grazing}$, respectively, were related to rainfall distribution. The general mean in crude protein content of forage was higher during the drier months. Average production of all grasses in this experiment was lower than in a similar experiment at Corozal in the humid region of Puerto Rico, the relative difference varied among cultivars.
\end{abstract}

\section{INTRODUCTION}

The soils of the northern coastal plains are characterized by being less acid than those of the humid mountain region (1). They range from gently sloping to sloping, have medium natural fertility and are very well suited for pastures. At the present time this area sustains the most important milkshed in Puerto Rico, accounting for more than 50\% of the total fresh milk produced. ${ }^{3}$

'Manuscript submitted to Editorial Board 30 April 1986.

${ }^{2}$ Associate Professor, Agronomy and Soils Department; Associate Agronomist (ret.), Corozal Substation; and Research Assistant, Corozal Substation, respectively, Agricultural Experiment Station, Mayagüez Campus, University of Puerto Rico, Río Piedras, P. R.

${ }^{3}$ Ramirez, A., 1984. Progress of the dairy industry in Puerto Rico. Presented at the Hill Farm Research Station, Louisiana State University, Homer, La. 71040. 
Digitaria pentzii Slenderstem PI 11537 and $D$. decumbens Transvala PR PI 6439 are highly productive grasses under cutting $(6,8,10,13)$. Both grasses have also produced high yields and persisted well for 2 years under intensive grazing $(3,10,15) . D$. milanjiana Pangola Soto is a highly productive grass in the humid mountain region when harvested by cutting (12), but it has not been evaluated for persistence under grazing.

Cynodon plectostachyus PR PI 11212, C. plectostachyus, PR PI 11487 and $C$. nlemfuensis var. nlemfuensis PR PI 2341 have been three of the most productive grasses under cutting management in different trials in the humid region $(7,8,11,14)$. In small plots $C$. dactylon Bermuda and $C$. nlemfuensis var. nlemfuensis produced high yields of forage and persisted under intensive grazing for 2 years in the mountain region (10). On the other hand, after 1 year of evaluation under clipping, C. dactylon cv. Coastcross-1 had been invaded by weeds (9).

Among Panicum maximum types, Guinea and USDA 259553 each produced over $40,000 \mathrm{~kg} / \mathrm{ha}$ of dry forage with intensive cutting management in Gurabo (5), whereas Makueni PR PI 12917 has been as productive as Guinea under high levels of fertilization in the humid mountain region at Corozal (13). Guinea is among the highest producers of dry matter under grazing management (17) and Makueni has been one of the outstanding grasses in the region under grazing in small plots (10). These outstanding grasses need further evaluation in different regions of Puerto Rico to determine their performance under the complexity of grazing conditions in different agroecosystems.

The objective of the present investigation was to evaluate forage production and persistence of selected grass species adapted to the humid northern coastal plains of Puerto Rico subjected to grazing in small plots.

\section{MATERIALS AND METHODS}

The experiment was conducted on a private farm located near Barceloneta, lat. $18^{\circ} 27^{\prime} \mathrm{N}$ and long. $66^{\circ} 33^{\prime} \mathrm{W}$, at an elevation of $4 \mathrm{~m}$, in the humid northern coastal plains of Puerto Rico. Mean annual rainfall is $1360 \mathrm{~mm}$, well distributed throughout the year, and mean daily temperature is $20^{\circ} \mathrm{C}$, with seasonal variations of about $2^{\circ} \mathrm{C}$.

The soil is an Islote clay (Typic Tropudalfs) with original pH 5.3, 80 $\mathrm{p} / \mathrm{m}$ available $\mathrm{P}$ (Bray I) and $81 \mathrm{p} / \mathrm{m}$ exchangeable $\mathrm{K}$ in th top $15 \mathrm{~cm}$. The soil was limed at a rate of $2,240 \mathrm{~kg} / \mathrm{ha} 3$ months before planting in May 1981 and 1 year later in May 1982.

A randomized block design with four replications and 10 grass cultivars (table 1) in 8x8 m plots was established August 1981. The grasses were fertilized with a 15-5-10 fertilizer at a rate of $1,600 \mathrm{~kg} / \mathrm{ha} /$ year divided in four equal applications throughout he year. 
All plots were cut initially at $10 \mathrm{~cm}$ above ground in October 1981, 2 months before grazing at 3- to 5-week intervals to a height of about 15 $\mathrm{cm}$, with a group of 35 adult animals for a period of 1 to 2 days (mob grazing).

The forage offered in each plot was determined by sampling before and after grazing, with the pair quadrats method described by Rivera and Rodríguez (2). Green forage weight was determined in the field, and samples were dried at $55^{\circ} \mathrm{C}$ in a forced air oven for 48 hours to determine dry matter content. Monthly hand-picked forage samples were composited by replicates of each grass treatment, dried at $55^{\circ} \mathrm{C}$ in a forced air oven, ground in a Wiley mill to pass a 1-mm screen and analyzed for $\mathrm{N}$ content with a Technicon Autoanalyzer. Crude protein (CP) was calculated as $\mathrm{N} \times 6.25$. The procedure was repeated over 1.5 consecutive years with data from 13 grazing dates during the first year (1981 to 1982) and 7 for the following 6 months (1983). The experiment was discontinued because of deterioration of one of the cultivars by overgrazing.

\section{RESULTS AND DISCUSSION}

Panicum maximum USDA PI 259553 was the most productive grass under grazing during the first year of experiment in 1981-1982, although it was not significantly different $(\mathrm{P}=0.05)$ from Cynodon plectostachyus Star, Cynodon sp. Local, Digitaria pentzii cv Slenderstem, C. nlemfuensis Star, $P$. maximum Makueni, and $D$. decumbens Transvala (table 2). Guinea and D. milanjiana Pangola Soto were the least productive cultivars $(P=0.05)$.

The average production of all grasses was related to rainfall distribution. The lowest average production per period for all grasses, $0.26 \mathrm{ton} / \mathrm{ha}$ (table 2), was from April 12 to May 10, which was a dry period in the

TABLE 1.-Identification of grass cultivars

\begin{tabular}{|c|c|c|c|}
\hline $\begin{array}{l}\text { Grass } \\
\text { number }\end{array}$ & Species & USDA PI ${ }^{1}$ & $\mathrm{PR} \mathrm{PI}^{2}$ \\
\hline 1 & Cynodon sp. cv. Local & - & - \\
\hline 2 & Digitaria pentzii cv. Slenderstem & 300985 & 11537 \\
\hline 3 & $\begin{array}{l}\text { C. nlemfuensis var. } \\
\text { nlemfuesis cv. Star }\end{array}$ & - & 2341 \\
\hline 4 & C. plectostachyus cv. Star & 341818 & 11487 \\
\hline 5 & C. dactylon cv. Coastcross- 1 & 255455 & 11504 \\
\hline 6 & Digitaria malanjiana & & \\
\hline & cv. Pangola Soto & 299699 & 6543 \\
\hline 7 & Panicum maximum cv. Guinea & - & - \\
\hline 8 & P. maximum cv. Guinea & 259553 & 3622 \\
\hline 9 & P. maximum cv. Makueni & 349676 & 12917 \\
\hline 10 & D. decumbens cv. Transvala & 299752 & 6439 \\
\hline
\end{tabular}

${ }^{1}$ United States Department of Agriculture plant introduction number.

'University of Puerto Rico Agricultural Experiment Station plant introduction number. 
TABLE 2.-Mean dry forage on offer of tropical grasses under grazing in the humid northern coastal plains at Barceloneta, Puerto Rico, 1981 -1982

Grazing dates

\begin{tabular}{|c|c|c|c|c|c|c|c|c|c|c|c|c|c|c|c|}
\hline $\begin{array}{l}\text { Grass } \\
\text { number }\end{array}$ & $\begin{array}{c}\text { Dec. } 1 \\
1981\end{array}$ & $\begin{array}{l}\operatorname{Jan} .4 \\
1982\end{array}$ & $\begin{array}{c}\text { Feb. } 4 \\
1982\end{array}$ & $\begin{array}{c}\text { Mar. } 8 \\
1982\end{array}$ & $\begin{array}{c}\text { Apr. 12 } \\
1982\end{array}$ & $\begin{array}{c}\text { May } 10 \\
1982\end{array}$ & $\begin{array}{c}\text { Jun. } 7 \\
1982\end{array}$ & $\begin{array}{c}\text { Jun. } 28 \\
1982\end{array}$ & $\begin{array}{c}\text { Aug. 2 } \\
1982\end{array}$ & $\begin{array}{c}\text { Aug, } 31 \\
1982\end{array}$ & $\begin{array}{c}\text { Sep. } 27 \\
1982\end{array}$ & $\begin{array}{c}\text { Nov. I } \\
1982\end{array}$ & $\begin{array}{c}\text { Nov. } 30 \\
1982\end{array}$ & $\begin{array}{l}\text { Mean per } \\
\text { grazing }\end{array}$ & $\begin{array}{l}\text { Total } \\
\text { per year }\end{array}$ \\
\hline \multicolumn{16}{|c|}{ ton/ha } \\
\hline 3 & $1.88 \mathrm{a}^{1}$ & $1.67 \mathrm{a}$ & $0.90 \mathrm{ab}$ & $0.91 \mathrm{abc}$ & $1.14 \mathrm{a}$ & $0.27 \mathrm{abc}$ & $1.59 \mathrm{ab}$ & $0.74 \mathrm{a}$ & $0.97 \mathrm{c}$ & $0.76 \mathrm{~cd}$ & $0.48 \mathrm{bcd}$ & $0.60 \mathrm{a}$ & $0.26 \mathrm{f}$ & $0.94 a b c$ & $12.21 \mathrm{abc}$ \\
\hline 2 & $1.77 \mathrm{ab}$ & $0.66 \mathrm{bc}$ & $0.87 \mathrm{ab}$ & $0.74 \mathrm{bc}$ & $1.40 \mathrm{a}$ & $0.34 \mathrm{ab}$ & $1.26 \mathrm{abc}$ & $0.93 \mathrm{a}$ & $1.39 \mathrm{bc}$ & $1,86 \mathrm{a}$ & $0.91 \mathrm{~b}$ & $0.75 \mathrm{a}$ & $0.48 \mathrm{ef}$ & $1.03 \mathrm{abc}$ & $13.36 \mathrm{abc}$ \\
\hline 5 & $1.74 \mathrm{ab}$ & $0.73 \mathrm{bc}$ & $0.34 \mathrm{~b}$ & $0.71 b c$ & $1.24 \mathrm{a}$ & $0.06 \mathrm{bc}$ & $1.32 \mathrm{abc}$ & $0.77 \mathrm{a}$ & $1.54 \mathrm{abc}$ & $0.87 \mathrm{bed}$ & $0.95 \mathrm{~b}$ & $0.64 \mathrm{a}$ & $0.65 \mathrm{de}$ & $0.99 \mathrm{bc}$ & $11.56 \mathrm{bc}$ \\
\hline 6 & $1.32 \mathrm{bc}$ & $1.03 \mathrm{~b}$ & $0.41 \mathrm{ab}$ & $1.00 \mathrm{abc}$ & $0.17 \mathrm{c}$ & 0.01 & $0.50 \mathrm{c}$ & $0.02 \mathrm{~b}$ & $0.32 \mathrm{c}$ & $0.13 \mathrm{~d}$ & $0.17 \mathrm{~s}$ & $0.00 \mathrm{~b}$ & $0.00 \mathrm{~g}$ & $0.39 \mathrm{~d}$ & $5.07 \mathrm{~d}$ \\
\hline 1 & $1.19 \mathrm{c}$ & $0.68 b c$ & $1.17 \mathrm{ab}$ & $1.21 \mathrm{ab}$ & $1.28 \mathrm{a}$ & $0.24 \mathrm{abc}$ & $0.93 b c$ & $0.74 \mathrm{a}$ & $0.72 \mathrm{abc}$ & $1.76 \mathrm{abc}$ & $0.24 \mathrm{~cd}$ & $0.37 \mathrm{ab}$ & $1.15 \mathrm{ab}$ & $0.97 a b c$ & $12.68 \mathrm{abc}$ \\
\hline 4 & $1.16 \mathrm{c}$ & $0.94 b c$ & $0.85 \mathrm{ab}$ & $0.99 \mathrm{abc}$ & $1.27 \mathrm{a}$ & $0.31 \mathrm{abc}$ & $215 \mathrm{a}$ & $0.99 \mathrm{a}$ & $1.92 \mathrm{abc}$ & $1.70 \mathrm{abc}$ & $0.63 \mathrm{bcd}$ & $0.47 \mathrm{a}$ & $0.86 \mathrm{bcd}$ & $1.10 \mathrm{ab}$ & $14.24 \mathrm{ab}$ \\
\hline 10 & $0.99 \mathrm{c}$ & $0.77 \mathrm{bc}$ & $1.12 \mathrm{ab}$ & $1.38 \mathrm{a}$ & $0.96 \mathrm{ab}$ & $0.27 \mathrm{abc}$ & $1.73 \mathrm{ab}$ & $0.88 \mathrm{a}$ & $0.75 \mathrm{c}$ & $1.70 \mathrm{abc}$ & $0.81 \mathrm{bc}$ & $0.44 a b$ & $1.01 \mathrm{bc}$ & $0.99 \mathrm{abc}$ & $12.91 \mathrm{abc}$ \\
\hline 9 & $0.34 \mathrm{~d}$ & $0.25 \mathrm{c}$ & $0.68 \mathrm{ab}$ & $0.73 \mathrm{bc}$ & $0.41 \mathrm{bc}$ & $0.32 \mathrm{abv}$ & $1.77 \mathrm{ab}$ & $0.74 \mathrm{a}$ & $2.34 \mathrm{ab}$ & $2.33 \mathrm{a}$ & $1.64 \mathrm{a}$ & $0.55 \mathrm{a}$ & 0.73 cde & $0.99 \mathrm{abc}$ & $12.83 \mathrm{abc}$ \\
\hline 8 & $0.25 \mathrm{~d}$ & $0.29 \mathrm{c}$ & $0.99 \mathrm{ab}$ & $0.88 \mathrm{abc}$ & $0.68 \mathrm{abc}$ & $0.48 \mathrm{a}$ & $1.88 \mathrm{a}$ & $0.94 \mathrm{a}$ & $2.79 \mathrm{a}$ & $2.06 \mathrm{a}$ & $1.57 \mathrm{a}$ & $0.70 \mathrm{a}$ & $1.38 \mathrm{a}$ & $1.15 \mathrm{a}$ & $14.98 \mathrm{a}$ \\
\hline 7 & $0.14 \mathrm{~d}$ & $0.38 \mathrm{bc}$ & $1.11 \mathrm{ab}$ & $0.52 \mathrm{c}$ & $0.31 b c$ & $0.23 \mathrm{abc}$ & $1.55 \mathrm{ab}$ & $0.73 \mathrm{a}$ & $2.60 \mathrm{ab}$ & $1.78 \mathrm{abc}$ & $0.62 \mathrm{bcd}$ & $0.31 \mathrm{ab}$ & $0.49 \mathrm{ef}$ & $0.83 \mathrm{c}$ & $10.79 \mathrm{c}$ \\
\hline Mean & 1.08 & 0.74 & 0.86 & 0.91 & 0.89 & 0.26 & 1.47 & 0.75 & 1.63 & 1.49 & 0.80 & 0.49 & 0.70 & 0.92 & 12.06 \\
\hline
\end{tabular}

${ }^{1}$ Means in the same column followed by the same letter do not differ significantly at the $5 \%$ probability level. 
TABLE 3.-Monthly rainfall at Barceloneta during the course of the experiment, 1981-1983

\begin{tabular}{lrrr}
\hline Month & 1981 & 1982 & 1983 \\
\hline & & $m m$ & \\
January & 42 & 92 & 15 \\
February & 98 & 61 & 9 \\
March & 48 & 35 & 22 \\
April & 108 & 35 & 218 \\
May & 440 & 282 & 126 \\
June & 170 & 98 & 119 \\
July 151 & 86 & 120 & \\
August & 139 & 72 & 116 \\
September & 132 & 124 & 77 \\
October & 90 & 63 & \\
December & 423 & 236 & 65 \\
Total & 1,991 & 1,331 & 1,111 \\
\hline
\end{tabular}

region in 1982 (table 3). P. maximum USDA PI 259553 produced almost twice as much forage during this dry period (table 2), although it was not statistically different $(\mathrm{P}=0.05)$ from the other grasses except $C$. dactylon Coastcross-1, and D. milanjiana Pangola Soto.

During the rainy period from May 10 to June 7, 1982, all grasses were highly productive, except for $D$. milanjiana Pangola Soto, which continued to deteriorate under the intensive grazing. During the second part of the rainy season, from August 2 through September 26, 1982, (fig. 1), P. maximum USDA PI 259553 and Makueni continued to be the most productive grasses and Cynodon Local and C. nlemfuensis Star the least productive $(\mathrm{P}=0.05)$. Total production for the first year of $P$. maximum USDA PI 259553 and Cynodon Local was higher than that of the other grasses $(\mathrm{P}=0.05)$, but production of the former was more consistent throughout the year (table 2).

In the final 6 months of the experiment, from January 3 to June 13 , 1983 (table 4), P. maximum USDA PI 259553 continued to be the most productive grass, although it was not significantly different $(\mathrm{P}=0.05)$ from $D$. decumbens Transvala, $C$. dactylon Coastcross-1 and D. pentzii. The production of $P$. maximum Makueni and C. plectostachyus Star was intermediate; $C$. nlemfuensis Star and Guinea were the least productive, and $D$. milanjiana Pangola Soto did not persist under intensive grazing $(\mathrm{P}=0.05)$.

Again, average production of all grasses was related to rainfall distribution. The exceptionally high production in January 3, 1983, (table 4), followed very high rainfall in December 1981 (fig. 1). Thereafter, production decreased markedly in January, February and March 1983, a period of low rainfall and cool weather. During this period $D$. decumbens Tran- 
TABLE 4.-Mean dry forage on offer of tropical grasses under grazing in the humid northern coastal plains at Barceloneta, Puerto Rico, 1983

\begin{tabular}{|c|c|c|c|c|c|c|c|c|c|}
\hline \multirow[b]{2}{*}{$\begin{array}{l}\text { Grass } \\
\text { number }\end{array}$} & \multicolumn{7}{|c|}{ Grazing dates } & \multirow[b]{2}{*}{$\begin{array}{l}\text { Mean per } \\
\text { grazing }\end{array}$} & \multirow[b]{2}{*}{$\begin{array}{c}\text { Total } \\
\text { per year }\end{array}$} \\
\hline & $\begin{array}{c}\text { Jan. } 3 \\
1983\end{array}$ & $\begin{array}{l}\text { Jan. } 24 \\
1983\end{array}$ & $\begin{array}{c}\text { Feb. } 24 \\
1983\end{array}$ & $\begin{array}{c}\text { Mar. } 29 \\
1983\end{array}$ & $\begin{array}{c}\text { May3 } \\
1983\end{array}$ & $\begin{array}{c}\text { May 23 } \\
1983\end{array}$ & $\begin{array}{c}\text { Jun. } 13 \\
1983\end{array}$ & & \\
\hline \multicolumn{10}{|c|}{ ton/ha } \\
\hline 10 & $3.02 \mathrm{a}^{4}$ & $1.68 \mathrm{a}$ & $1.13 \mathrm{ab}$ & $1.25 \mathrm{ab}$ & $1.45 \mathrm{bcd}$ & $0.17 b c$ & $1.11 \mathrm{~b}$ & $1.40 \mathrm{a}$ & $9.08 \mathrm{a}$ \\
\hline 8 & $2.74 \mathrm{ab}$ & $0.41 \mathrm{~cd}$ & $0.40 \mathrm{~cd}$ & $0.93 \mathrm{abc}$ & $2.56 \mathrm{a}$ & $0.77 \mathrm{ab}$ & $2.12 \mathrm{a}$ & $1.43 \mathrm{a}$ & $10.01 \mathrm{a}$ \\
\hline 9 & $2.72 \mathrm{ab}$ & $0.30 \mathrm{~cd}$ & $0.37 \mathrm{~cd}$ & $0.34 \mathrm{bcd}$ & $1.64 \mathrm{~b}$ & $0.81 \mathrm{ab}$ & $1.45 \mathrm{ab}$ & $1.09 \mathrm{bed}$ & $7.63 \mathrm{bcd}$ \\
\hline 5 & $2.42 \mathrm{ab}$ & $0.88 \mathrm{bc}$ & $1.46 \mathrm{a}$ & $1.09 \mathrm{abc}$ & $1.32 \mathrm{bed}$ & $0.51 \mathrm{abc}$ & $1.22 \mathrm{~b}$ & $1.27 \mathrm{ab}$ & $8.88 \mathrm{ab}$ \\
\hline 2 & $2.33 \mathrm{ab}$ & $1.06 \mathrm{abc}$ & $1.24 \mathrm{ab}$ & $1.38 \mathrm{a}$ & $1.11 \mathrm{~cd}$ & $0.42 \mathrm{abc}$ & $0.60 \mathrm{bc}$ & $1.16 \mathrm{bc}$ & $8.15 \mathrm{abc}$ \\
\hline 4 & $1.93 \mathrm{ab}$ & $1.34 \mathrm{ab}$ & $0.90 \mathrm{abc}$ & $1.05 \mathrm{abc}$ & $1.31 \mathrm{bcd}$ & $0.40 \mathrm{abc}$ & $0.79 \mathrm{bc}$ & $1.10 \mathrm{bed}$ & $7.72 \mathrm{bcd}$ \\
\hline 3 & $1.69 \mathrm{~b}$ & $0.76 \mathrm{bed}$ & $0.73 \mathrm{bc}$ & $0.49 \mathrm{abc}$ & $0.70 \mathrm{e}$ & $0.70 \mathrm{e}$ & $0.23 \mathrm{abc}$ & $0.77 \mathrm{e}$ & $5.43 \mathrm{e}$ \\
\hline 7 & $1.65 \mathrm{~b}$ & $0.23 \mathrm{~cd}$ & $0.45 \mathrm{~cd}$ & $0.18 \mathrm{~cd}$ & $1.57 \mathrm{~b}$ & $0.95 \mathrm{a}$ & $0.75 \mathrm{bc}$ & $0.83 \mathrm{de}$ & $5.79 \mathrm{de}$ \\
\hline 1 & $1.51 \mathrm{~b}$ & $0.73 \mathrm{bcd}$ & $0.78 \mathrm{bc}$ & $1.33 \mathrm{a}$ & 0.96 cde & $0.13 \mathrm{bc}$ & $1.20 \mathrm{~b}$ & $0.95 \times \mathrm{de}$ & 6.64 cde \\
\hline 6 & $0.00 \mathrm{c}$ & $0.00 \mathrm{e}$ & $0.00 \mathrm{e}$ & $0.00 \mathrm{e}$ & $0.00 f$ & $0.00 \mathrm{~d}$ & $0.00 \mathrm{~d}$ & $0.00 \mathrm{f}$ & $0.00 \mathrm{f}$ \\
\hline Mean & 2.01 & 0.74 & 0.75 & 0.81 & 1.26 & 0.44 & 1.01 & 1.00 & 7.00 \\
\hline
\end{tabular}

'Means in the same columm followed by the same letter do not differ significantly at the $5 \%$ probability level.

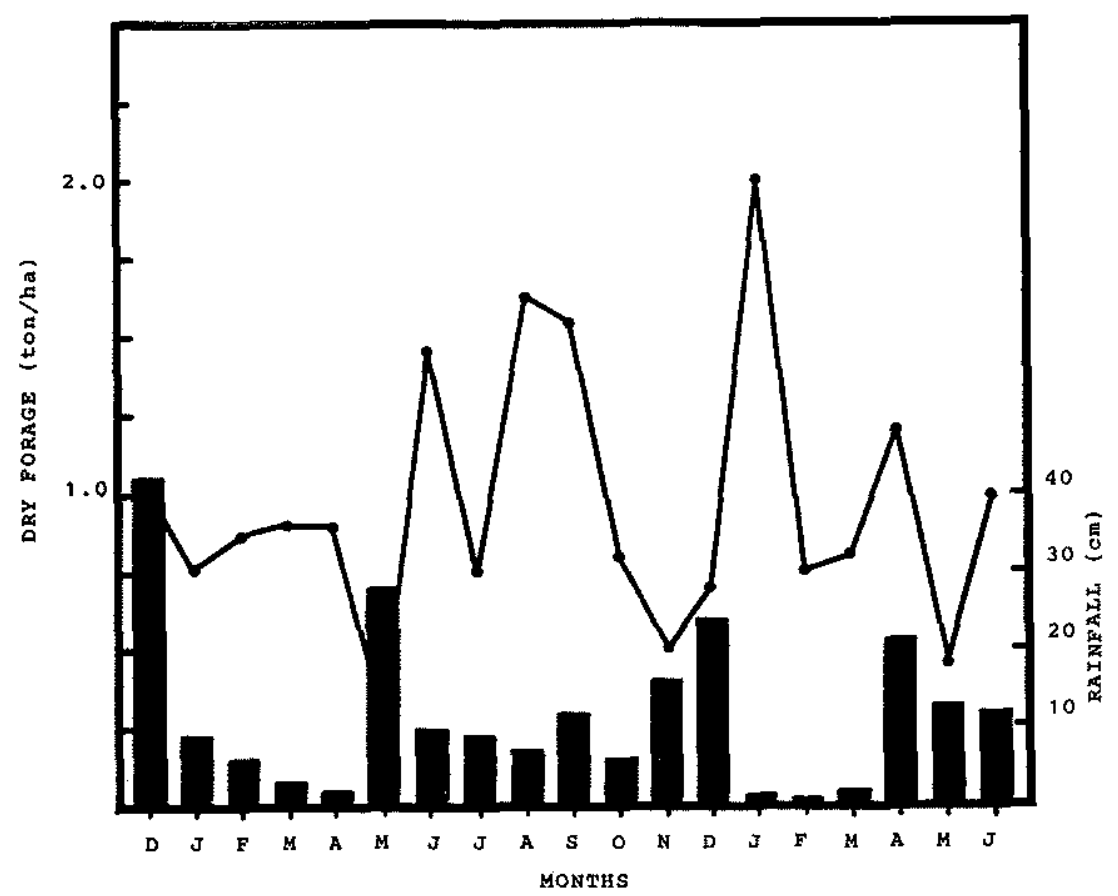

FIG. 1. - Seasonal dry forage on offer, mean for all grasses grazed in small plots every 3 to 5 weeks at Barceloneta, Puerto Rico, 1981 to 1983. 
vala, C. dactylon Coastcross-1, D. pentzii Slenderstem, and C. plectostachyus Star were in general more productive than Guinea grass, unlike during the rainy season after March 29, 1983. The experiment was terminated after the June 13, 1983, grazing because of low production and deterioration of the stand in some plots. In general, production of all grasses in this experiment was lower than that observed in a similar experiment at Corozal, in the humid mountain region of Puerto Rico (9). This can be ascribed to lower production during the dry season at Barceloneta, but it varied among cultivars. For instance, the production of $P$. maximum Makueni was only $60 \%$ and that of $C$. nlemfuensis Star $80 \%$ of the production the one reported at Corozal, respectively (10); however, the production of C. plectostachyus was similar, and that of $D$. pentzii Slenderstem was higher in this experiment than the one at Corozal (10).

The mean CP content was similar for all grasses (table 5). C. plectostachyus, C. nlemfuensis var. nlemfuensis and the local cultivar of Cynodon sp. showed the higher CP content, $15.19,14.52$ and $14.57 \%$, respectively. The lowest CP content was found in $D$. pentzii cv. Slenderstem. In general, there was a tendency for a higher CP content associated with lower yields, and viceversa, except in November. There is a concentration or dilution effect, related to the amount of forage produced with similar $\mathrm{N}$ content in the soil which has been reported in previous experiments in the humid mountain region at Corozal $(4,16,17)$.

Rainfall distribution is better and the dry season is shorter in the humid mountain region than in the northern coastal plains, and additional contribution factors may include differences in ambient temperatures during the dry season and in soil fertility. Nevertheless, $P$. maximum USDA PI 259553 is a cultivar highly productive in a number of different circumstances, i.e., under cutting in Gurabo (5), under grazing at Corozal (16) and as in the present investigation.

\section{RESUMEN}

Producción de gramíneas tropicales pastadas en diferentes ecosistemas agrícolas de Puerto Rico. II. Llanuras húmedas de la Costa Norte

En agosto de 1981 se estableció un experimento cerca de Barceloneta en las llanuras húmedas de la costa norte de Puerto Rico para determinar la producción y persistencia de 10 gramíneas tropicales pastadas en parcelas pequeñas. Cynodon local, Digitaria pentzii slenderstem, $C$. nlemfuensis estrella, C. plectostachyus estrella, C. dactylon coastcross-1, D. milaniiana pangola Soto, Panicum maximum guinea común, P. maximum USDA PI 259553, $P$. maximum makueni y $D$. decumbens Transvala se pastaron a intervalos de 3 a 5 semanas con un grupo de 35 animales adultos por un período de 1 a 2 días a una altura de $15 \mathrm{~cm}$. a partir de diciembre de 1981 hasta junio de 1983. P. maximum USDA PI 259553 fue la más productiva en 13 apacentamientos el primer año de 1981 a 1982, con una media de 
TABLE 5.-Mean crude protein content of dry forage on offer of tropical grasses under grazing in the humid northern coastal plains at Barceloneta, Puerto, Rico, 1981-1983

\begin{tabular}{|c|c|c|c|c|c|c|c|c|c|c|c|c|c|}
\hline \multirow[b]{2}{*}{$\begin{array}{l}\text { Grass } \\
\text { number }\end{array}$} & \multicolumn{12}{|c|}{ Grazing dates } & \multirow[b]{2}{*}{$\begin{array}{l}\text { Mean per } \\
\text { grazing }\end{array}$} \\
\hline & Dec. & Jan. & Feb. & Mar. & Apr. & May & Jun. & Jul. & Aug & Sept. & Oct. & Nov. & \\
\hline 4 & 20.75 & 17.62 & 15.50 & 16.75 & 9.87 & 18.37 & 14.25 & 11.75 & 15.87 & 14.12 & 15.37 & 12.12 & 15.19 \\
\hline 3 & 20.00 & 17.87 & 16.37 & 18.87 & 8.62 & 20.00 & 14.25 & 11.25 & 16.25 & 11.62 & 10.37 & 8.75 & 14.52 \\
\hline 5 & 19.62 & 14.25 & 12.00 & 13.25 & 10.25 & 20.00 & 12.00 & 10.00 & 15.25 & 9.62 & 10.62 & 10.50 & 13.11 \\
\hline 6 & 18.75 & 20.12 & 15.00 & 14.12 & 11.00 & 19.50 & 14.00 & 12.12 & 15.50 & 12.00 & 10.62 & 12.12 & 14.57 \\
\hline 9 & 18.75 & 14.37 & 13.12 & 11.62 & 11.12 & 24.12 & 9.87 & 7.37 & 13.37 & 10.50 & 10.75 & 8.25 & 12.77 \\
\hline 7 & 18.00 & 15.00 & 12.12 & 12.87 & 6.75 & 23.37 & 14.87 & I2.62 & 16.12 & 9.37 & 11.25 & 13.00 & 13.78 \\
\hline 8 & 17.62 & 14.25 & 11.00 & 12.62 & 11.62 & 20.87 & 13.20 & 12.40 & 13.62 & 10.75 & 9.62 & 13.12 & 13.39 \\
\hline 2 & 16.50 & 16.87 & 12.62 & 9.37 & 10.37 & 20.75 & 11.87 & 11.62 & 11.37 & 7.87 & 8.37 & 8.00 & 12.13 \\
\hline 1 & 15.00 & 16.62 & 15.00 & 17.25 & 14.25 & 17.00 & 13.37 & 11.19 & 14.25 & 14.12 & 15.00 & 11.37 & 14.57 \\
\hline Mean & 18.33 & 16.33 & 13.64 & 14.08 & 10.43 & 20.44 & 13.07 & 11.19 & 14.62 & 11.10 & 11.33 & 9.92 & 13.78 \\
\hline
\end{tabular}

'Means of 4 replications for two years. 
1.15 ton./ha. de materia seca por intervalo de apacentamiento aunque no fue estadísticamente diferente $(P=0.05)$ de Cynodon plectostachyus estrella, Cynodon sp. local, Digitaria pentzii slenderstem, $C$. nlemfuensis estrella, $P$. maximum makueni, y $D$. decumbens transvala. La guinea común y $D$. milaniiana pangola Soto fueron las menos productivas $(P=0.05)$. La producción media de todas las gramíneas estuvo relacionada con la distribución de las Iluvias; la mínima por período para todas las gramíneas, 0.26 ton./ha., ocurrió entre abril 12 y mayo 10, que correspondió a la época más seca en la región ese año. P. maximum USDA PI 259553 produjo casi el doble que las otras forrajeras durante este período seco, aunque no fue significativamente diferente $(P=0.05)$ de las otras, con excepción de $C$. dactylon coasteross-1 y $D$. milaniiana pangola Soto. Durante el período más lluvioso, de mayo 10 a junio 7, 1982, todas las gramíneas fueron altamente productivas, excepto D. milaniiana Pangola Soto, la cual continuó deteriorándose y no resistió el apacentamiento intensivo. El contenido medio en proteína bruta del forraje fue mayor durante los meses más secos. Durante 6 meses del segundo año, de enero 3 a junio 13, 1983, P. maximum USDAPI 259553 continuó siendo la más productiva, aunque no fue significativamente diferente $(P=0.05)$ de $D$. decumbens transvala, $C$. dactylon coastcross-1 y $D$. pentzii slenderstem. La producción de $P$. maximum makueni y $C$. pectostachyus estrella fue intermedia; $C$. nlemfuensis estrella y guinea común fueron las menos productivas $(P=0.05)$ y $D$. milaniiana pangola Soto no persistió con un apacentamiento intensivo. De nuevo, la producción media de todas las gramíneas estuvo relacionada con la distribución de las lluvias. La producción excepcionalmente alta en enero 3 de 1983, estuvo relacionada con fuertes lluvias en diciembre de 1982 . Luego la producción disminuyó marcadamente en enero, febrero y marzo de 1983, a causa de las pocas lluvias y posiblemente a temperaturas más bajas. Durante este período $D$. decumbens transvala, $C$. dactylon coastcross-1, $D$. pentzii slenderstem y $C$. plectostachyus estrella fueron en general más productivas que la guinea, a diferencia del caso durante la estación lluviosa después del 29 de marzo de 1983. El experimento se terminó en junio 13, de 1983 debido a la baja producción y deterioro de algunas parcelas. La producción media de todas las gramíneas en este experimento fue más baja que la observada en un experimento similar en Corozal en la región montañosa húmeda de Puerto Rico por la menor producción durante la estación seca, pero estas diferencias relativas variaron con las cultivares estudiadas.

\section{LITERATURE CITED}

1. Acevedo, G., 1982. Survey of Arecibo area of northern Puerto Rico. USDA Soil Conservation Service.

2. Rivera, E. and J. Rodríguez, 1980. Forage yield of five grasses under intensive grazing management in the humid region of Puerto Rico, J. Agric. Univ. P. R. 64 (3): 259-63.

3. Rodríguez, J., E. Rivera and J. Vicente-Chandler, 1980. Productivity of four intensively managed grasses under grazing management in the humid hill region of Puerto Rico, J. Agric. Univ. P. R. 64 (2): 236-46. 
4. Soldevila, M., 1980. Effect of rotation length in pangola pastures upon the liveweight gain of growing Holstein heifers, J. Agric. Univ. P. R. 64 (2): 243-46.

5. Sotomayor-Ríos, A., A. Acosta-Matienzo and J. Vélez-Fortuño, 1971. Yield comparison and plant character correlations of 16 Panicum accessions, J. Agric. Univ. P. R. 55 (2): 174-83.

6. - - - - and $-\frac{1}{-}$ 1973. Evaluation of seven forage grasses at two cutting stages, J. Agri, Univ, P, R. 57 (3): 173-8.

7. —, F. J. Juliá and J. A. Arroyo-Aguilú, 1974. Effects of harvest intervals on the yield and composition of 10 forage grasses, J. Agric. Univ. P. R. 58 (4): 448-55.

8. - J. Vélez-Santiago, S. Torres-Rivera and S. Silva, 1976. Effect of three harvest intervals on yield and composition of nineteen forage grasses in the humid mountain region of Puerto Rico, J. Agric. Univ, P. R. 60 (3): 294-309.

9. Tergas, L. E., J. Vélez-Santiago and D. Vera de Saldaña, 1986. Response of tropical grasses to potassium fertilization in the humid coastal region of Puerto Rico, $J$. Agric. Univ. P. R. 72 (1): 00.

10. $—-\ldots$, and -1986 . Productivity of tropical grasses under grazing in different agroecosystems in Puerto Rico. I Humid mountain, J. Agric. Univ. P. R. 72 (1): 00.

11. Vélez-Santiago, J. and J. A. Arroyo-Aguilú, 1983. Nitrogen fertilization and cutting frequency, yield and chemical composition of five tropical grasses, J. Agric. Univ. P. R. 67 (2): $61-69$.

12. - and - 1984. Comparison of six Stylosanthes eultivars and Digitaria milanjiana in the humid mountain region of Puerto Rico, J. Agric. Univ. P. R. 68 (4): 355-64.

13. — and - 1984. Influence of two fertilizer levels on forage and crude protein yields of seven tropical grasses, J. Agric. Univ. P. R. 68 (4): 471-78.

14. - A. Sotomayor-Ríos and S. Torres-Rivera, 1979. Effects of three harvest intervals and two fertilizer rates on the yield and HCN content of ten Cynodon cultivars, $J$. Agric. Univ. P. R. 63 (1): 35-44.

15. Vicente-Chandler, J, J. Rodríguez and F. Abruña, 1972. Effects of two heights and three intervals of grazing on the productivity of heavily fertilized Pangola grass pastures, J. Agric. Univ. P. R. 56 (2): 110-14.

16. — , R. Caro-Costas, F. Abruña y S. Silva, 1983. Producción y utilización intensiva de las forrajeras en Puerto Rico, Esta. Exp. Agrie. Univ. P. R. Bol. 271.

17. —, F. Abruna, R. Caro-Costas, J. Figarella, S. Silva and R. W. Pearson, 1974. Intensive Grassland Management in the Humid Tropics of Puerto Rico, Agric. Exp. Stn. Univ. P. R. Bull. 283. 